\title{
Réhabilitation esthétique et fonctionnelle par PACIU dans un cas de classe II squelletique
}

\section{Aesthetic and functional rehabilitation of a case of skeletal classe II by complete immediate denture}

\section{Résumé}

\section{MOTS-CLEFS :}

- Prothèse immédiate d'usage, classe II squelettique, prothèse amovible complète, re

\section{KEYWORDS:}

- Compete immediate denture, complete denture, skeletal Class II, tissular preparation

$\operatorname{AOS} n^{\circ} 280-2016$
La prothèse amovible complète immédiate d'usage (PACIU) est une alternative thérapeutique de choix pour aider le patient à surmonter les difficultés psychologiques et physiologiques liées au passage à l'édentation complète. Elle permet en effet un rétablissement immédiat de l'esthétique et des différentes fonctions orales et constitue un véritable guide de cicatrisation ostéo-muqueuse post-extractionnelle. Dans les cas de classe II squelettique, aux difficultés classiques de la PACIU, relatives ò l'enregistrement des surfaces dento-osteo-muqueuses (dualité tissulaire), à la simulation des extractions dentaires et à l'absence de tout essayage, se surajoute la problématique de la gestion occlusale de ce genre de réhabilitation au stade de l'enregistrement du rapport maxillo-mandibulaire et du montage des dents prothétiques. Les auteurs se proposent de montrer par le biais d'un cas clinique la démarche thérapeutique à adopter dans le traitement de ces situations cliniques par PACIU.
Abstract

The complete immediate denture is a treatment that can help the patient to overcome the psychological and physiological challenges associated with the transition to a full edentulous state. It offers an immediate restoration of aesthetics and different oral functions and a good quality of bone and mucosal healing. Achieving complete immédiat denture in cases of skeletal Class II has many difficulties relating to the registration of dental and osteo - mucosal surfaces (tissue duality), simulation of extractions and the lack of test of the anterior teeth is added the problem of occlusal management this kind of rehabilitation at the stage of registration of the maxilo- mandibular jaw relation and the montage of the prosthetic teeth's. The authors intend to show the therapeutic approach to be adopted in the treatment of these clinical situations by complete immediat denture.

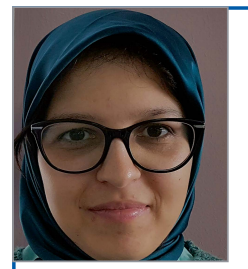

- Fadwa GUESSOUS DOSS, Résidente en prothèse adjointe - Service de Prothèse Adjointe, Université Mohamed V, Faculté de médecine dentaire de Rabat, BP 6212,

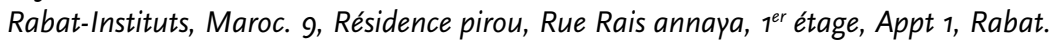
Tél. : o674528180, mail :dr.f.guessous@gmail.com

Leila FAJRI, Professeur agrégé en prothèse adjointe. Service de Prothèse Adjointe, Université Mohamed V, Faculté de médecine dentaire de Rabat, BP 6212, Rabat-Instituts, Maroc.

Nadia MERZOUK, Professeur d'Enseignement supérieur, Chef du service de Prothèse Adjointe Service de Prothèse Adjointe - Université Mohamed V, Faculté de médecine dentaire de Rabat, BP 6212, Rabat-Instituts, Maroc. 


\section{INTRODUCTION}

La prothèse amovible complète immédiate d'usage est une prothèse définitive confectionnée avant la séance des dernières extractions et insérée immédiatement après. Ce type de prothèse est préconisé pour assurer un passage moins traumatisant de l'édentement partiel à l'édentement complet [1].

C'est un choix thérapeutique intéressant lorsqu'il n'est pas possible de conserver les dents naturelles sur l'arcade à cause des lésions parodontales sevères ou de l'évolution carieuse importante [2].

L'élaboration de la PACIU requiert un protocole clinique rigoureux. En effet, elle doit répondre aux impératifs esthétiques et fonctionnels de la prothèse complète conventionnelle tout en surmontant les difficultés spécifiques de la prothèse immédiate $[3,4]$. À travers un cas clinique, les auteurs se proposent de décrire le protocole de gestion thérapeutique d'une réhabilitation maxillaire par PACIU chez un patient en classe II squelettique.

\section{OBSERVATION CLINIQUE}

Mr H M, agée de 64 ans, en bon état de santé général, consulte au service de prothèse adjointe pour une réhabilitation prothétique esthétique et fonctionnelle. L'anamnèse révèle un patient très affécté par la perte de ses dents suite à la maladie parodontale et notamment par la perte récente de la 11.

IL'examen exobuccal montre une diminution de l'étage inférieur de la face et un profil convexe (Fig. 1).

\section{L'examen endobuccal :}

- Au niveau maxillaire : Il révèle la persistance de 15 ; $12 ; 21$ et 22 ayant des atteintes parodontales sevères. Les crêtes édentées sont recouvertes d'une fibromuqueuse ferme et adhérente (Fig. 2).

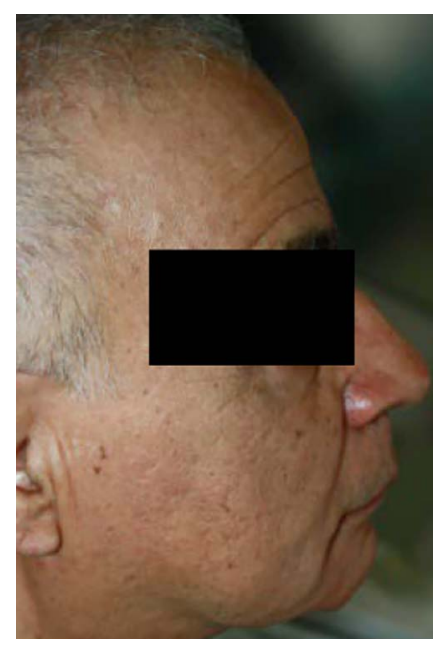

$\triangleleft$ Fig. 1 : Profil convexe avec léger affaissement de la DVO.

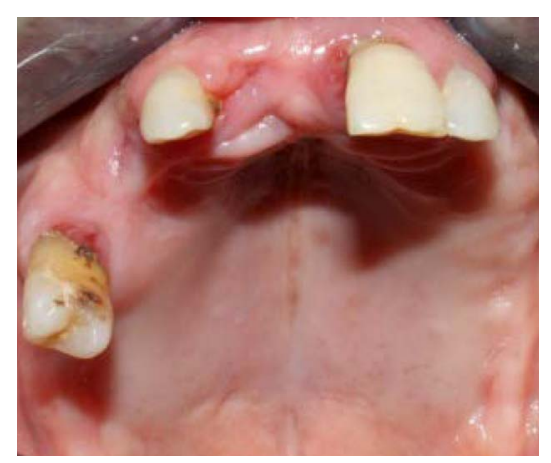

$\triangle$ Fig. 2 : Vue endo-buccale de la surface d'appui maxillaire.
- Au niveau mandibulaire : On note un édentement complet avec une résorption osseuse moyenne et une hyperplasie muqueuse au niveau du fond du vestibule antérieur en rapport avec un bord prothétique surétendu de son ancienne prothèse instable et défectueuse (Fig. 3a et b).

I L'examen radiologique montre une lyse osseuse horizontale importante dépassant le 1/3 apical des racines (Fig. 4 et 5).
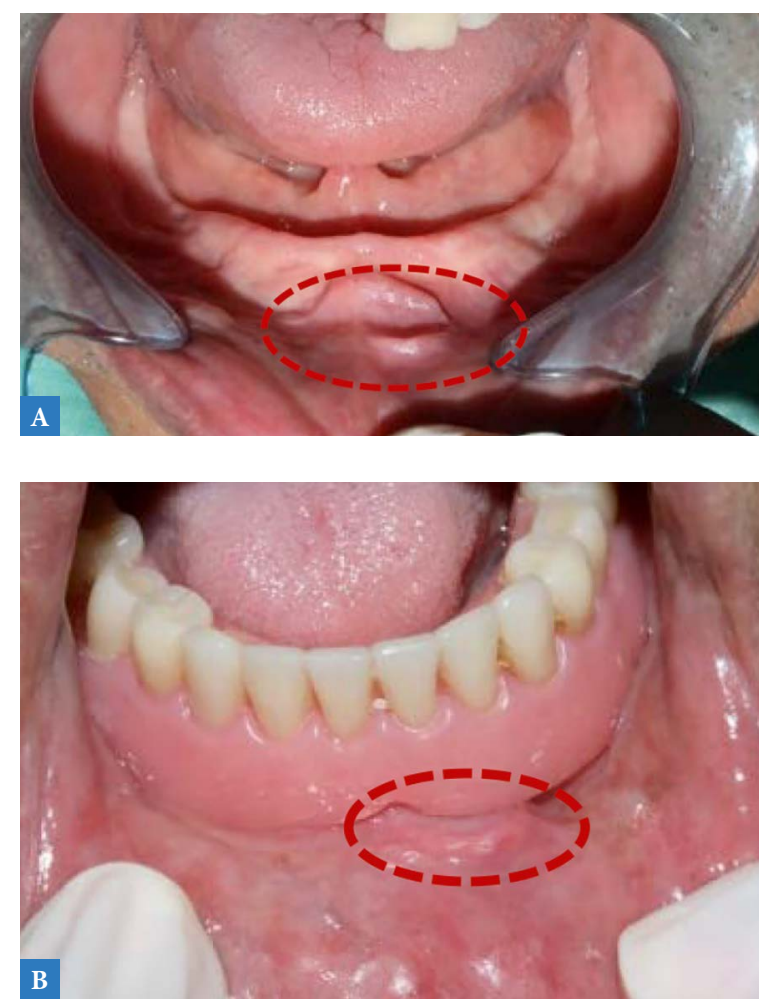

$\triangle$ Fig. 3a, b : Hyperplasie muqueuse en rapport avec le bord prothétique sur étendu d'une ancienne PAC inférieure.

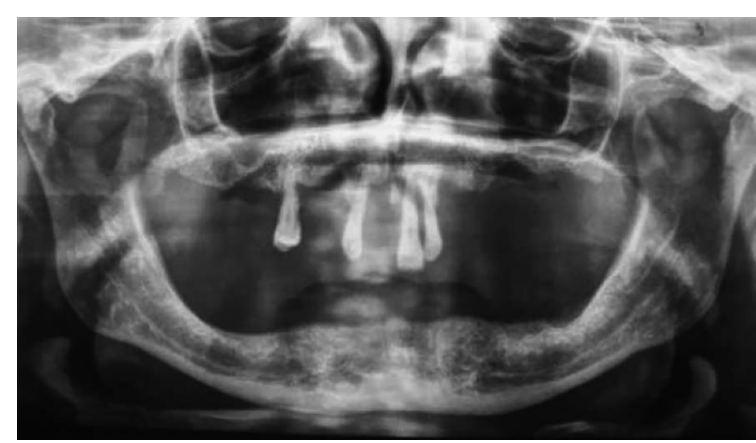

$\triangle$ Fig. 4 : Radiographie panoramique.

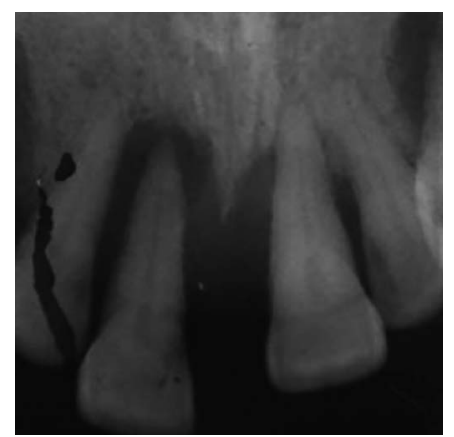

$\measuredangle$ Fig. 5 : Radiographie rétro-alvéolaire. 


\section{PROBLÉMATIQUE}

On distingue de nombreuses contraintes spécifiques à la situation clinique en rapport avec :

I Le contexte psychologique : traumatisme psychologique du patient dû à la perte de ses dents et à la condamnation de ses dents restantes en raison de la lyse osseuse terminale.

I Le contexte anatomique défavorable de l'arcade maxillaire : les tubérosités sont effacées; le palais est plat; les crêtes sont peu hautes avec des insertions freinales et ligamentaires hautes.

I Le rapport inter-arcade défavorable de classe II squelettique : ce dernier compromet l'équilibre biomécanique, en effet le décalage inter-arcade aboutira à un montage avec un surplomb important entre les groupes incisivo-canins maxillaire et mandibulaire favorisant une interposition nocive et inesthétique de la lèvre inférieure dans le vide ainsi créé au niveau incisivo-canin.

\L'arcade antagoniste édentée totale marquée par une résorption assez importante et la présence d'une hyperplasie muqueuse au niveau du fond du vestibule antérieur ce qui rend la stabilité prothétique précaire.

\section{DÉCISION THÉRAPEUTIQUE}

Compte tenu des éléments de diagnostic précités et des objectifs thérapeutiques escomptés, nous avons opté pour la réalisation de :

I Une PACIU au niveau maxillaire : afin de répondre aux exigences esthétiques et psychologiques du patient, guider la cicatrisation post-extractionnelle et limiter la résorption osseuse.

I Une nouvelle prothèse amovible complète au niveau mandibulaire répondant aux impératifs de stabilité, rétention et intégration occlusale. Elle va servir par ailleurs à réaliser une mise en condition tissulaire visant à traiter l'hyperplasie muqueuse.

\section{DÉMARCHE THÉRAPEUTIQUE}

Elle suit trois phases essentielles pré-prothétique, prothétique et post-prothétique conduisant à l'élaboration et à la maintenance d'une réhabilitation prothétique de qualité.

\section{Phase pré-prothétique}

Elle constitue un préalable indispensable à la réalisation prothétique. Elle comprend :

I un assainissement dento-parodontal visant à diminuer l'inflammation ; $\checkmark$ l'extraction de la 15 afin d'obtenir des surfaces d'appui postérieures stables avec une bonne cicatrisation osseuse gage d'une fiabilité de l'enregistrement ultérieur du rapport maxillo-mandibulaire.

\section{Phase prothétique proprement dite Empreintes primaires}

Ces étapes doivent être effectuées avec soin de manière à éviter l'extraction prématuré des dents mobiles. Elles sont réalisées à l'aide d'alginate. Des portes empreintes de type Rim-Lock ${ }^{\circledast}$ sont utilisés en vue d'enregistrer la totalité des surfaces d'appui y compris le fond de vestibule en regard des dents, la morphologie, la situation et la position des dents résiduelles maxillaires [5].

\section{Empreintes anatomo-fonctionnelles}

\section{Au niveau maxillaire}

Elle a un triple objectif :

$\checkmark$ la gestion de la dualité tissulaire ;

$\checkmark$ l'enregistrement du joint antérieur (présence des dents);

I'enregistrement de l'ensemble de la surface d'appui avec un joint periphérique étanche.

Elle est réalisée à l'aide d'un porte empreinte individuel confectionné à partir d'un modèle primaire. $\mathrm{Vu}$ la mobilité des dents et la présence de zones rétentives, ce dernier est conçu sous forme d'un PEI fenestré en regard des dents, associé à un couvercle démontable (Fig. 6). Ce type de porte empreinte présente l'avantage d'assurer un confort du patient lors de l'enregistrement physiologique du joint périphérique, et un démoulage de l'empreinte sans fracture des dents après coulée du modèle $[5,6]$. Elle est réalisée en trois étapes :

$\checkmark$ Réalisation du joint périphérique : il est effectué en regard du secteur édenté dans un premier temps à l'aide d'un matériau thermoplastique type pâte de Kerr ${ }^{\oplus}$. Dans un second temps, en regard $d u$

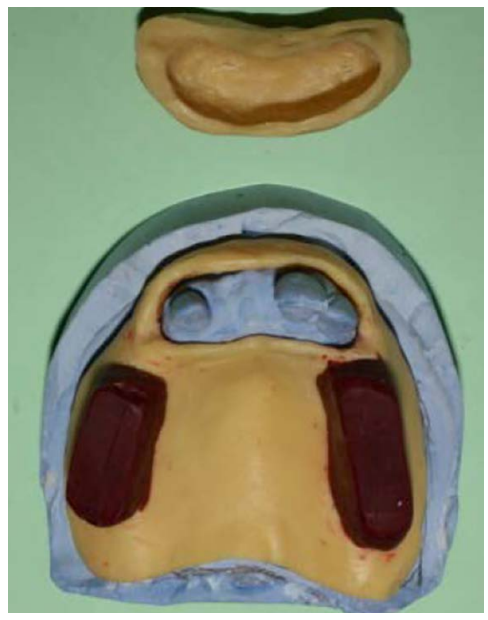

$\triangle$ Fig. 6 : PEI avec une ouverture operculée par un couvercle. 
secteur denté antérieur, un matériau élastique qui est le polyéther type Imprégum ${ }^{\circledR}$ est nécessaire pour l'obtention d'un joint souple (Fig. 7) [5, 7].

I Une empreinte de stabilisation au niveau des structures ostéo-muqueuses est effectuée à l'aide de la pâte d'oxyde de zinc (Impression past ${ }^{\varpi}$ ) pour enregistrer un enfoncement physiologique de la fibro-muqueuse sous faible pression digitale (Fig. 8).

$\checkmark$ une empreinte globale anatomo-fonctionnelle : Un matériau élastique de moyenne viscosité type polysulfure (Permlastic Regular ${ }^{\circledR}$ ) est disposé dans le couvercle. Le matériau de faible viscosité (Permalstic Light ${ }^{\oplus}$ ) est injecté au niveau des dents et placé au niveau du PEI en regard du secteur édenté. Cette empreinte globale permet d'enregistrer la différence de dépréssibilité tissulaire (Fig. 9) [8].

Au niveau mandibulaire : Une empreinte secondaire classique est réalisée en deux étapes : un remarginage à la pâte de kerr ${ }^{\circledast}$ et un surfaçage à la pâte d'oxyde de zinc avec une exploitation maximale des surfaces d'appui.

\section{Rapport maxillo-mandibulaire (RMM)}

Le rapport maxillo-mandibulaire est enregistré puis transféré sur articulateur, en se servant

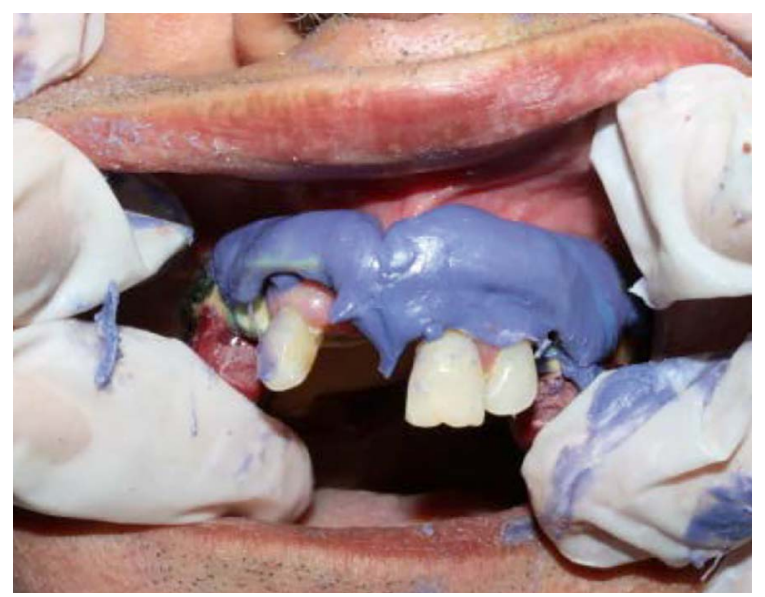

$\triangle$ Fig. 7 : Joint souple au niveau de la région antérieure à l'impregum .

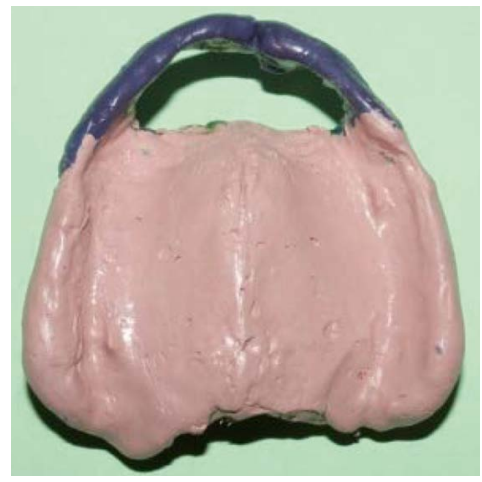

Fig. 8 : Stabilisation du PEI à l'Impression past ${ }^{\oplus}$.



Fig. 9 : Empreinte de la surface d'appui et des dents réalisée avec du Permlastic Light ${ }^{\circledast}$. de bases d'occlusion minces et résistantes [5] en résine auto-polymérisable comportant des bourrelets en stent's remplaçant les dents absentes selon les séquences suivantes (Fig. 10a et b) [9].

I Détermination de la situation idéale du bord libre des incisives supérieures (dents antérieures égréssées) selon les critères esthétiques et phonétiques (Fig. 11a).

> Orientation du plan d'occlusion parallèlement au plan bi-pupillaire antérieurement (Fig. 11b) et au plan de Camper postérieurement (Fig. 11c), sans que la règle de Fox ne s'appuie sur les dents naturelles afin d'éviter toute erreur liée à la différence de dualité tissulaire entre les dents naturelles et la fibro-muqueuse qui recouvre les surfaces d'appui édentées.

I Établissement de la DVO physiologique à partir de la DV de repos. Dans notre cas nous n'avons pas eu besoin d'augmenter la DVO puisque l'arcade inférieure est édentée complète.

Les modèles sur articulateur mettent en évidence un décalage antéro-postérieur important entre les arcades maxillaire et mandibulaire établissant un rapport analogue à celui d'une classe II squelettique. (Fig. 12).
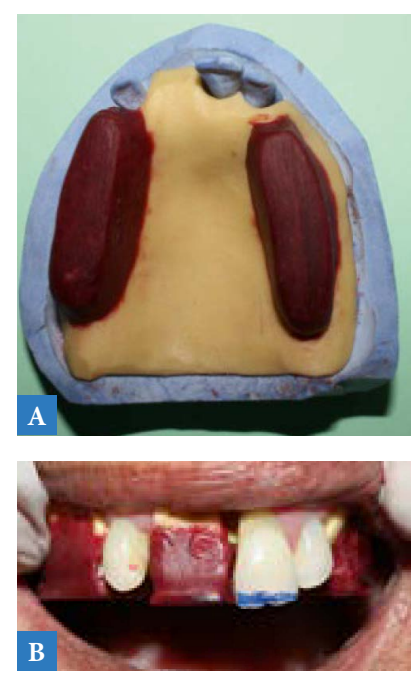

$\measuredangle$ Fig. 10a, b : Support d'enregistrement du rapport intermaxillaire.
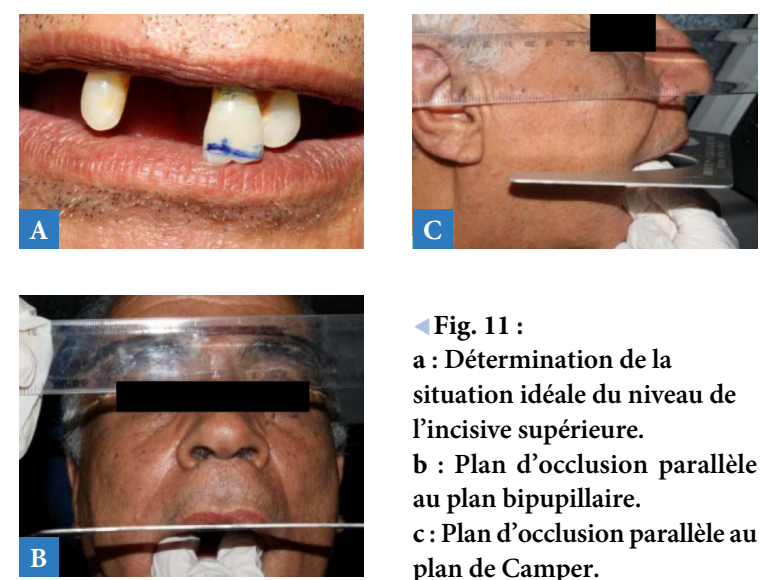

Fig. 11 :

a : Détermination de la situation idéale du niveau de l'incisive supérieure.

b : Plan d'occlusion parallèle au plan bipupillaire.

c: Plan d'occlusion parallèle au plan de Camper. 


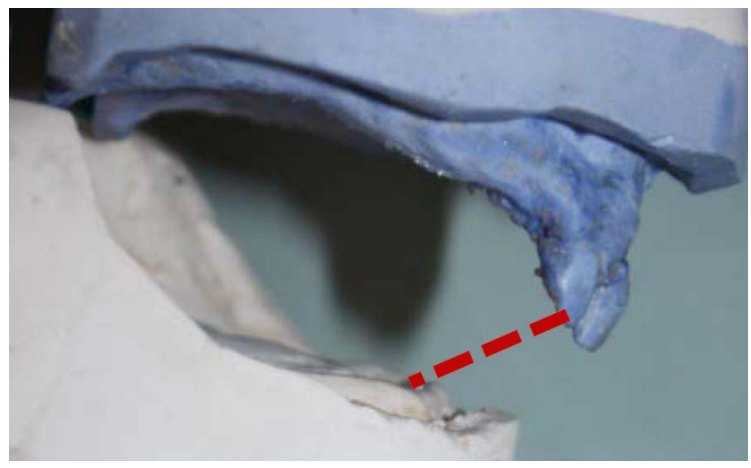

Choix, montage des dents prothétiques et essayage esthétique et fonctionnel

La situation du point inter-incisif et la ligne du sourire sont reportées sur la maquette et la dent restante suivant les mêmes critères que la prothèse totale.

Le choix des dents prothétiques est guidé par les dents antérieures persistantes sur l'arcade en termes de forme, de teinte et de dimensions (Fig. 13).
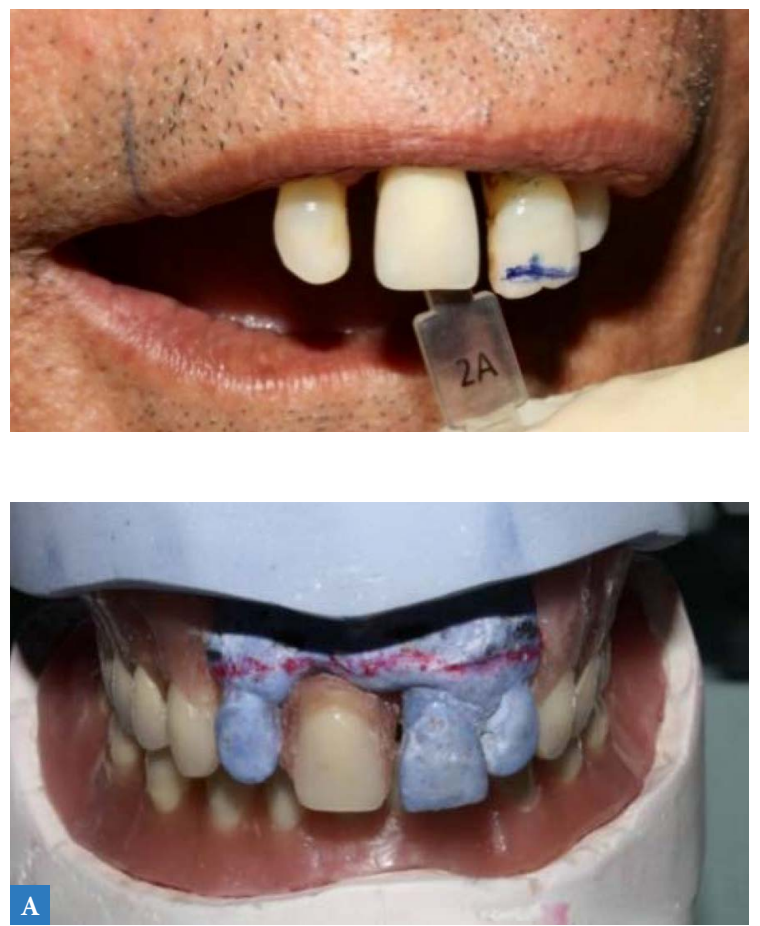

$\triangle$ Fig. 12 : Une rétrognathie mandibulaire importante.

Le montage des dents postérieures est réalisé en premier lieu, il obéit aux règles classiques de montage en prothèse complète à savoir le centrage des forces à l'intérieur des surfaces d'appui, le respect couloir prothétique, le respect du plan d'occlusion et de la stabilité occlusale (Fig. 14a) [10, 12].

\section{Essayage des dents prothétiques}

L'essayage des secteurs postérieurs supérieurs en confrontation avec le montage complet inférieur a permis la vérification du RMM préalablement enregistré, ainsi que le plan d'occlusion.

Par ailleurs, l'essayage de la 11 nous a permis en outre de valider la teinte, la forme et surtout la position de la dent par rapport au plan sagittal médian et dans le sens antéro-postérieur pour diminuer le décalage inter-arcade et apprécier le degré de redressement par rapport à la 21 persistante (Fig. 14b).

\section{Clés en silicone}

Avant l'extraction des dents du modèle, deux clés en silicone de haute viscosité sont réalisées : La première est une clé vestibulaire des dents antérieures restantes (Fig. 15a) et la deuxième est un mordu occlusal (Fig. 15b). Elles constituent un repère pour le montage définitif des dents prothétiques grâce à l'enregistrement de la situation des dents résiduelles et du point inter incisif ainsi que du montage postérieur essayé et validé en bouche $[5,12,13]$.

$\checkmark$ Fig. 13 : Choix des dents prothétiques à partir des dents résiduelles.

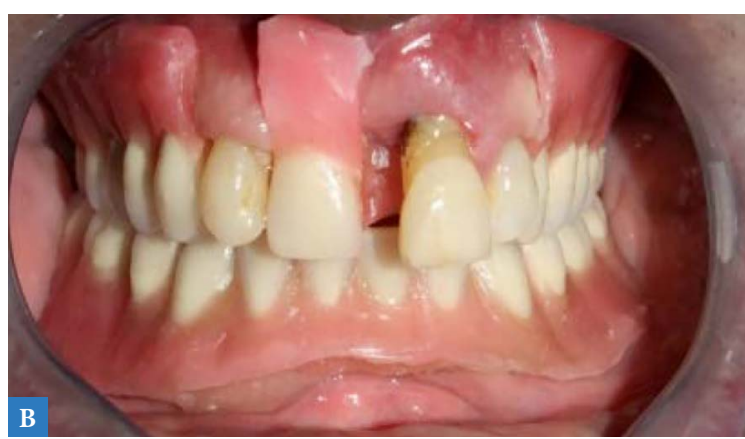

$\triangle$ Fig. 14 : Montage et essayage des dents prothétiques avant l'extraction des dents résiduelles du modèle.
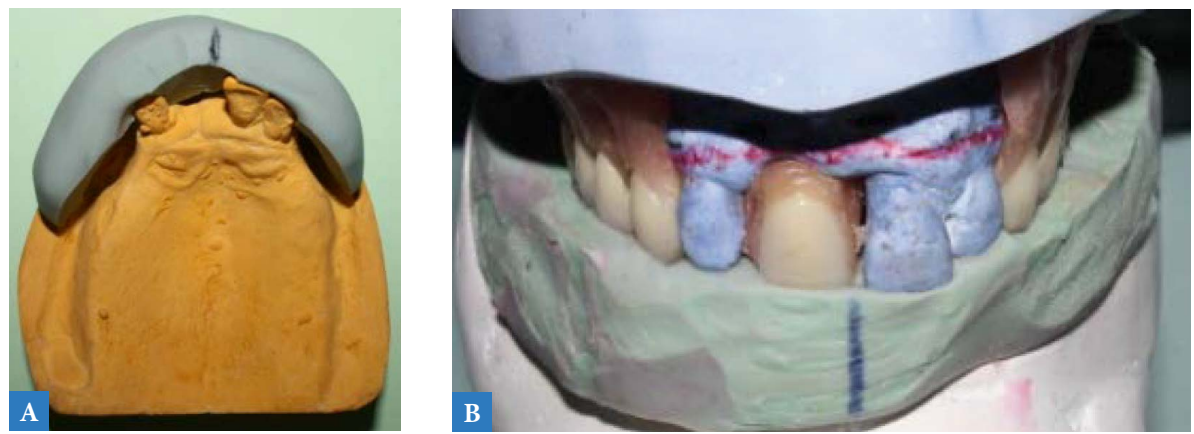

Fig. 15a, b :

Clés en silicone

pour l'enregistrement des repères. 


\section{Extraction des dents et aménagement de la crête en plâtre}

La régularisation osseuse simulée sur le modèle en plâtre doit être non mutilante pour maintenir suffisamment de capital osseux compte tenu de la résorption au maxillaire.

Néanmoins, lors de cette manœuvre, il est nécessaire de tenir compte de divers paramètres à savoir: la profondeur des poches parodontales évaluée cliniquement, la quantité d'os résiduel évaluée radiologiquement (Fig. 16), l'épaisseur de la fibro-muqueuse ainsi que la perte de substance liée à la cicatrisation et à la chirurgie correctrice (Fig. 17) $[15,16]$.

\section{Montage complet des dents prothétiques}

Le montage antérieur est réalisé en utilisant les références fournies par les clés en silicone (Fig. 18a et b). Cependant, le surplomb dû au décalage inter-arcade affectant l'équilibre biomécanique des prothèses doit être réduit par une vestibulo-version des incisives inférieures (dans les limites autorisées par le couloir prothétique) et un montage plus reculé des incisives supérieures par rapport aux incisives naturelles (Fig. 19) offrant ainsi l'épaisseur nécessaire à la fausse gencive.

\section{Temps chirurgical et pose de la prothèse}

Le guide chirurgical est préparé au laboratoire en résine transparente à partir du duplicata du modèle secondaire maxillaire après élimination des dents $\mathrm{du}$
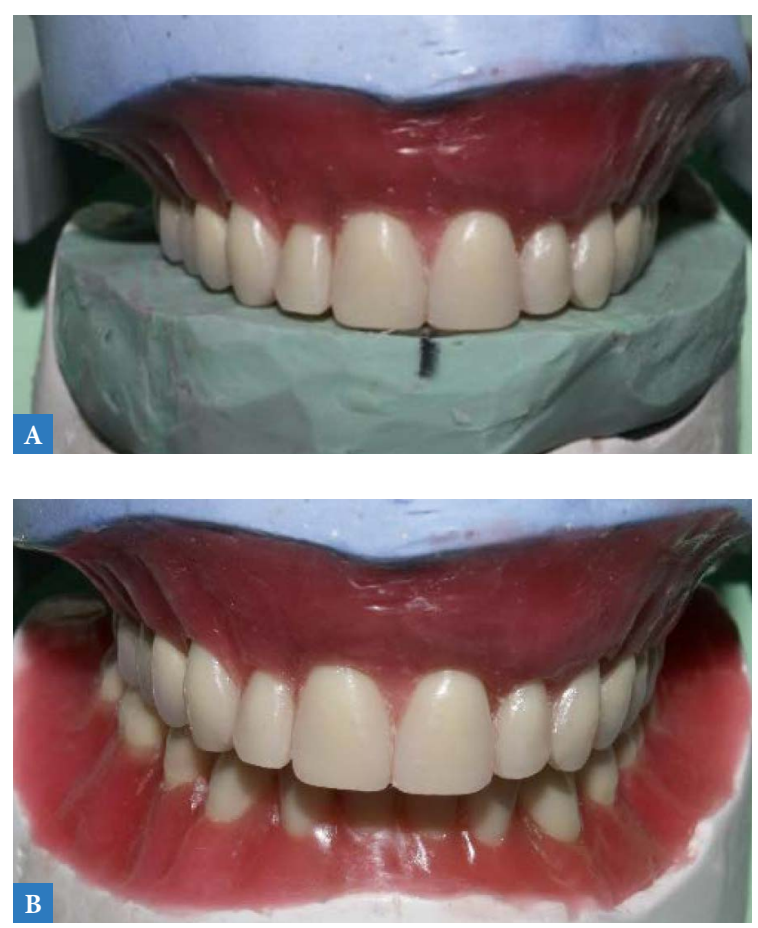

$\triangle$ Fig. 18 : Montage complet qui respecte les paramètres enregistrés.

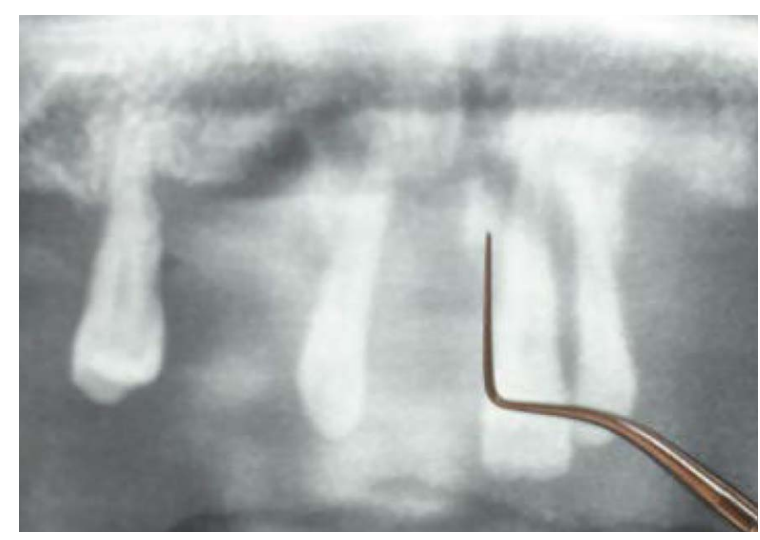

$\triangle$ Fig. 16 : Évaluation radiologique de la profondeur des poches parodontales.

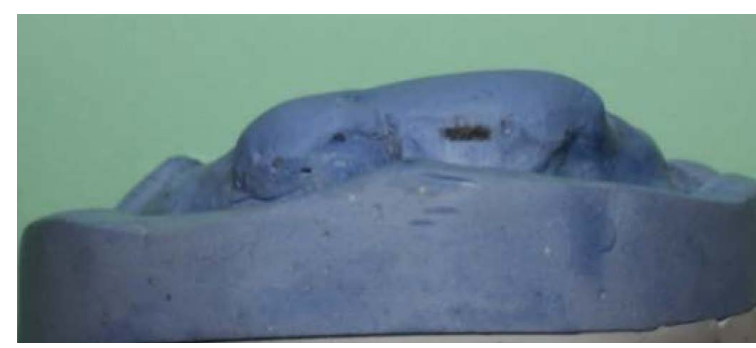

$\triangle$ Fig. 17 : Préparation du modèle après l'extraction des dents.

plâtre (Fig. 20). Après extraction des dents (Fig. 21a et $\mathbf{b}$ ), ce guide chirurgical permet de visualiser les zones de compression (Fig. 22), et les corrections des rebords osseux sont réalisées (Fig. 23) exactement de la même façon que sur le modèle en plâtre pour obtenir une adaptation parfaite de la prothèse d'usage aux surfaces d'appui $[8,17]$.

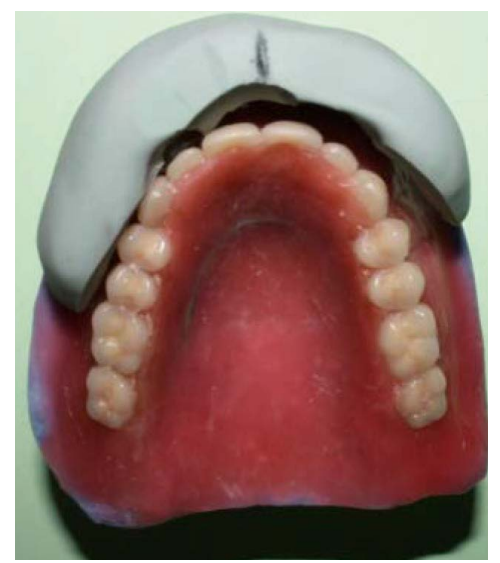

$<$ Fig. 19 : Incisives reculées par rapport aux incisives naturelles.

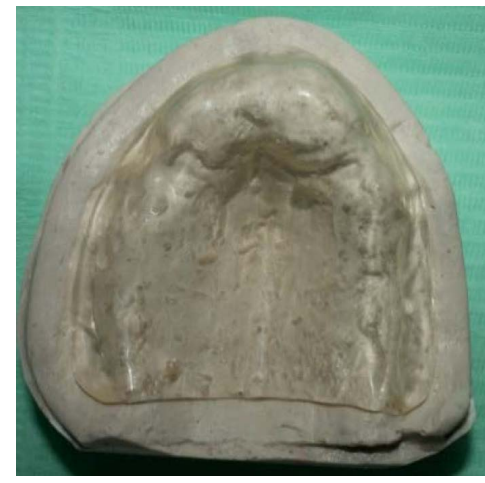

$\succ$ Fig. 20 : Guide chirurgical en résine transparente réplique exacte de la base prothétique. 

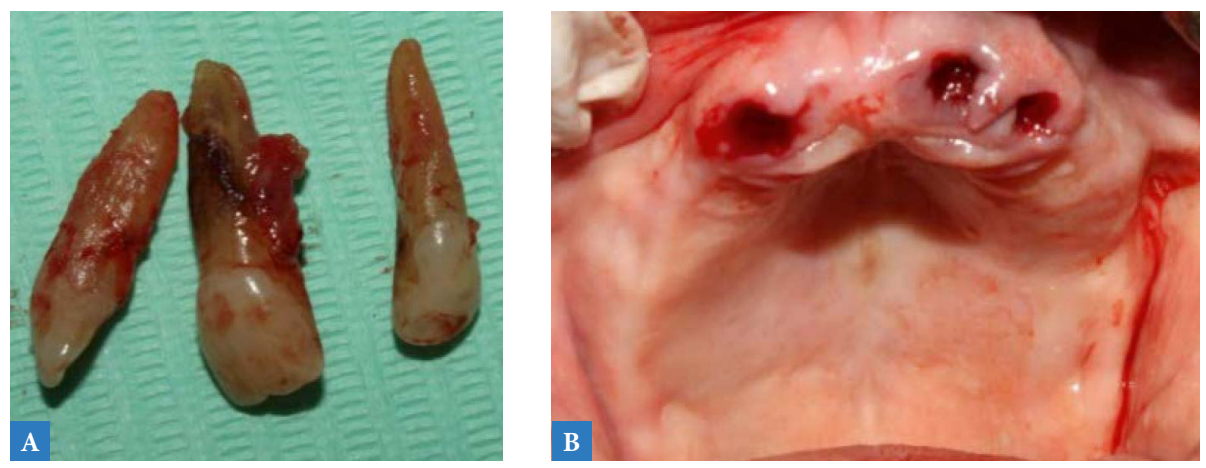

Fig. 21 : Extraction de 12,21 et 11 .

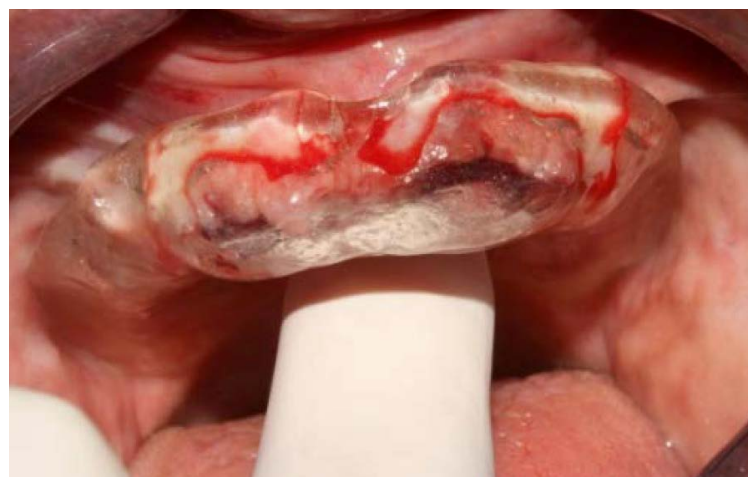

$\triangle$ Fig. 22 : Mise en évidence des zones de compression de la fibromuqueuse par le guide chirurgical.

\section{Insertion des prothèses en bouche} (Fig. 24, 25 et 26)

Le patient est invité à serrer ses dents pendant 10 minutes sur deux rouleaux de coton pour placer la prothèse à fond sur ses surfaces d'appui et éviter l'œè̀me post-opératoire avant de vérifier l'occlusion en relation centrée.

Il sera avisé de ne pas retirer sa prothèse pendant 48 heures de façon à éviter les difficultés de réinsertion

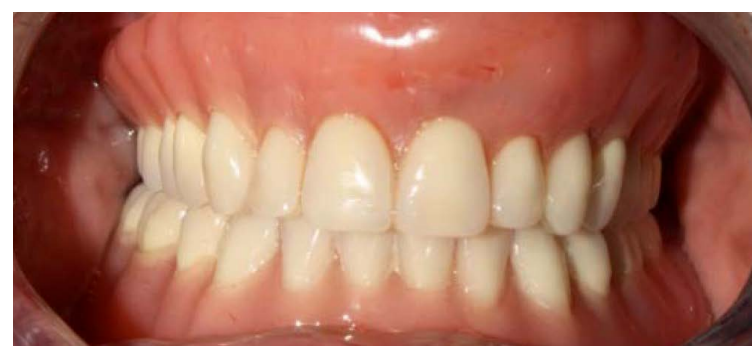

$\triangle$ Fig. 24 : Insertion de la PACIU et vérification des contacts occlusaux.

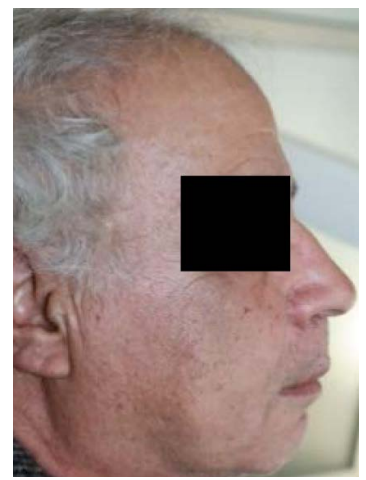

Fig. 25 : Vue de profil : Rétablissement de la DVO et du soutien labial.

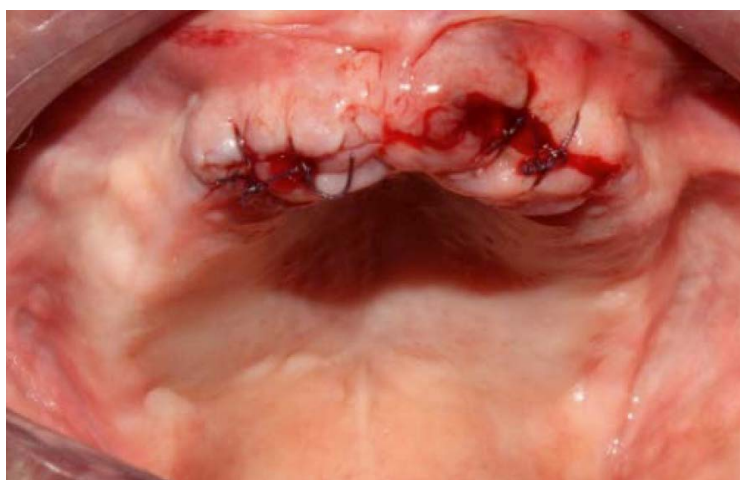

$\triangle$ Fig. 23 : Réalisation des sutures après régularisation osseuse.

qui peuvent survenir à cause de l'œdème. Par la suite, l'adaptation de la base est vérifiée et l'équilibration occlusale est effectuée tout au long des séances de contrôle.

\section{Mise en condition tissulaire}

au niveau mandibulaire

Une mise en condition tissulaire est réalisée en utilisant la nouvelle prothèse pour supporter le conditionneur (Fitt de kerr ${ }^{\circledR}$ ) (Fig. 27). Ce dernier est renouvelé

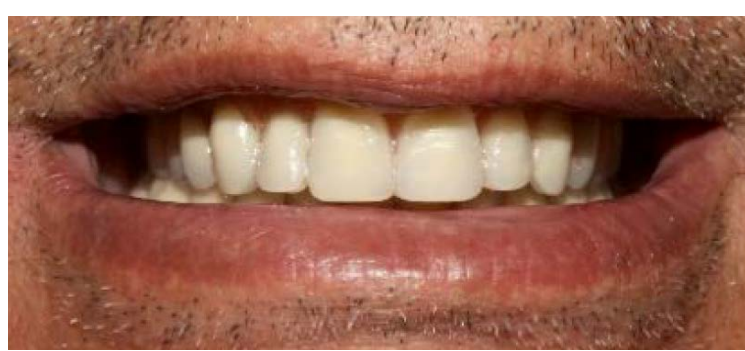

$\triangle$ Fig. 26 : Sourire de satisfaction du patient.



$<$ Fig. 27 : Prothèse inférieure garnie de conditionneur tissulaire. 
toutes les semaines. Deux mois après, une nette amélioration est constatée (Fig. 28) et la prothèse est ensuite rebasée.

Par ailleurs, nous constatons que la cicatrisation ostéo-muqueuse post-extractionnelle guidée par la PACIU est optimale (Fig. 29).

\section{DISCUSSION}

Bien qu'il existe de nombreuses limites liées à la PACIU, ce type de prothèse définitive offre au patient une possibilité thérapeutique intéressante du point de vue esthétique et surtout psychologique garantissant un passage graduel sans heurt de l'édentement partiel à l'édentement total.

Cependant, le risque de déception du patient par le résultat esthétique n'est pas écarté vu l'absence de l'essayage du montage des dents antérieures. Néanmoins, le sens clinique du praticien, l'analyse esthétique du cas, le respect des repères esthétiques et leur matérialisation au moyen de maquettes et de clés permet de sursoir à cet inconvénient.

La PACIU offre en outre une cicatrisation osseuse de qualité favorisant l'organisation rationnelle des trabécules osseuses.

Bien que la prothèse immédiate reste une solution de choix dans certaines situations cliniques, elle requiert des compétences spécifiques aux différentes phases de réalisation et plus particulièrement lors de l'étape des empreintes. Le choix approprié du type de PEI et des matériaux à empreintes et le respect de la technique sont indispensable à la réussite de ces dernières.

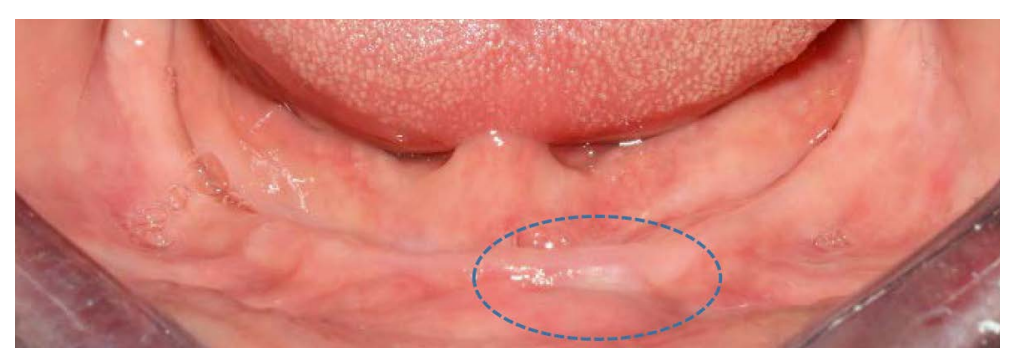

$\triangle$ Fig. 28 : Une nette amélioration de la lésion après deux mois de mise en condition tissulaire.
Les séances d'équilibration et de maintenance peuvent être nombreuses et prolongées mais pourtant indispensables pour garantir l'intégration de la prothèse à moyen et à long terme.

En raison de ces contraintes et de la coopération nécessaire du patient pour mener à bien ce genre de traitement, il est important de sélectionner les cas appropriés d'un point de vue psychologique et de bien expliquer au patient la nature et difficulté du travail avant le début du traitement afin d'obtenir leurs consentement éclairé.

\section{CONCLUSION}

Dans notre cas, la gestion thérapeutique par PACIU nous a permis d'obtenir un rendu esthétique très apprécié par le patient, de favoriser l'intégration psychologique de la prothèse sans passer par une phase d'édentation complète source d'angoisse pour le patient. Sur le plan équilibre occlusal, les artifices de montage employés ont permis de réduire l'intensité du décalage inter-arcade dans le cadre d'une occlusion intégralement équilibrée respectée.

Enfin, la gestion globale du cas par le biais de la mise en condition tissulaire au niveau mandibulaire, la régularisation osseuse post-extractionnelle raisonnée et contrôlée au moyen du guide chirurgical et la cicatrisation osseuse guidée par PACIU, sont des facteurs qui nous ont permis d'optimiser la qualité du tissu ostéo-muqueux constituant la surface d'appui, la préservation du capital osseux et la majoration de l'équilibre de la prothèse bi-maxillaire complète.

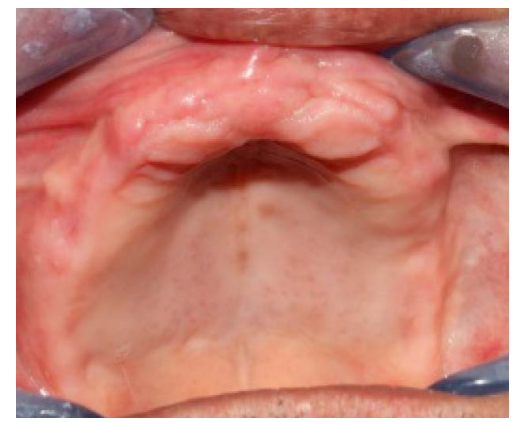

$<$ Fig. 29 : L'arcade maxillaire un mois après la chirurgie. 


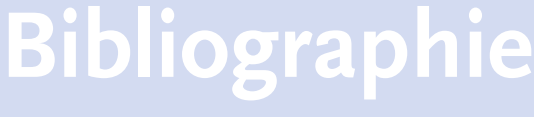

[1] Kraljevie S, and Coll. Immediate Complete Denture. Acta Stomat Croat 2001; Vol35; 281-285.

[2] Viennot S, Moyencourt C, Millet C, Buch D. Réhabilitation esthétique et fonctionnelle par prothèse complète immédiate : étapes cliniques. Cah Prothèse 2004;126:9-18.

[3] Smith RA. Immediate complete dentures- A starting point. J Am Dent Assoc 1993 ; 87:641-645.

[4] Pompignoli M, Postaire M, Raux D. Clinical succes in immedate complete dentures: An alternative approch.Hanover Park : Quintessence International, 2008.

[5] Berteretche MV, Hüe O. Prothèse complète immédiate. Encycl Med Chir (Elsevier SAS, Paris) Odontologie 2005 ; 23-325-K-10.

[6] Rignon-Bret $C$, Rignon-Bret JM. Prothèse immédiate d'usage. In : Prothèse amovible complète, prothèse immédiate, prothèse supraradiculaire et implantaire. Rueil-Malmaison : $C d P$, 2002:135-166.

[7] Herbout B, Postaire M. Prothèse complète immédiate d'usage. Actualisation de la technique. Cah Prothèse 2000;111:55-65.
[8] Abdelkoui A, Fajri L, Benamar A, Abdedine A. La prothèse complète immédiate d'usage : Réalisation temps par temps. Clinic 2013; Vol 34 : 87-95.

[9] Rignon-Bert JM. Determination du rapport intermaxillaire en prothèse complète immédiate ( ${ }^{\mathrm{e}}$ partie). Inf Dent 1989, $36: 3367-3378$.

[10] Schoendorff R, Jeanin C. Prothèse immédiate. Paris, Elsevier, Encycl Méd Chir Odontologie 1998; 23-325-K-10:1-10.

[11] Berteretche $M-V$, Hue $O$. La prothèse complète immédiate : une entité clinique, mais différentes approches. Cah Proth 1998; 104:89-101.

[12] Hüe O, Berteretche MV. Prothèse complète. Réalité clinique - solutions thérapeutiques. Paris : Quintessence, 2004.

[13] Janati G, Belmekhannate S. Intégration esthétique de la prothèse immédiate et des empreintes à visée esthétique. Le courrier du dentiste ; Janvier 2012. http://www.lecourrierdudentiste.com/ dossiers-du-mois/integration-esthetiquede-la-prothese-complete-interet-de-laprothese-immediate-et-des-empreintes-a-viseeesthetique.html
[14] Rignon-Bert C, Rignon-Bert JM. Traitement d'un cas de dysharmonie occlusale par prothèse immédiate amovible. Alternatives $1999 ; 3: 9-17$.

[15] Rignon-Bret JM, Martineau C. Prothèse complète immédiate. La rectification du modèle, l'étape chirurgicale et la pose de la prothèse. Inf Dent 1990;7:489-497.

[16] Rodney D, and coll. Cast modification for immediate complete dentures: Traditional and contemporary considerations with an introduction of spatial modeling. J Prosthet Dent 2008;100:399-405.

[17] Abdelkoui A, Fajri L, Benamar M, Abdedine A. Le guide chirurgical en prothèse complète immédiate d'usage. Web J Dent 2011 ; 6,3:6-11.

[18] Jivanescu A, Marcauteanu C, Bratu D. Immediate complete denture: A case report. TMJ 2003, Vol. 53, No. 3-4: 293-296.

[19] Regragui A, Abdedine A, Sefrioui, Merzouk N. Les causes d'echec en prothèse amovible complète d'usage. WebJDent; $\mathrm{Vol} 2 \mathrm{~N}^{\circ} 4$.

[20] Dales B, Daas M, Rignon-Bert JM. Restauration par prothèse amovible complète immédiate unimaxillaire d'usage. Méthodologie pour une situation clinique fréquente. Clinic $2005 ; 26: 243-258$. 